this association was lost among children with active infection (adj. OR $1.59,95 \%$ CI 0.72 to 3.54 ).

Conclusions The association between markers of atopy and wheeze increased with age. The results suggest modification of the relationship between SPT and wheeze by active geohelminth infections.

\section{P2-198 OVERWEIGHT ASSOCIATED WITH NON-ATOPIC WHEEZE IN RURAL TROPICS}

doi:10.1136/jech.2011.142976j.32

\begin{abstract}
${ }^{1,2} \mathrm{~A}$ L Moncayo, ${ }^{*}{ }^{1} \mathrm{M}$ Vaca, ${ }^{1} \mathrm{~S}$ Erazo, ${ }^{1} \mathrm{G}$ Oviedo, ${ }^{1} \mathrm{I}$ Quinzo, ${ }^{1} \mathrm{M}$ E Chico, ${ }^{3} \mathrm{~T}$ A E PlattsMills, ${ }^{2} \mathrm{~S}$ Alvim, ${ }^{4} \mathrm{~L}$ C Rodrigues, ${ }^{2} \mathrm{M}$ L Barreto, ${ }^{1,5} \mathrm{P}$ J Cooper. ${ }^{1}$ Universidad San Francisco de Quito, Colegio de Ciencias de la Salud, Quito, Ecuador; ${ }^{2}$ Universidade Federal da Bahia, Instituto de Saúde Coletiva, Salvador, Bahia, Brazil; ${ }^{3}$ University of Virginia, Asthma and Allergic Diseases Center, Virginia, USA; ${ }^{4}$ London School of Hygiene and Tropical Medicine, Department of Epidemiology, London, UK; ${ }^{5}$ Liverpool School of Tropical Medicine, Liverpool, UK
\end{abstract}

Introduction The parallel rise in prevalence of asthma and overweight/obesity in some Latin American countries has led to suggestions of a link between the two epidemics. The aim of this study was to explore the effects of being overweight on wheeze, exercise-induced bronchospasm and atopy.

Methods A case-control study was conducted among 809 AfroEcuadorian children aged 7-19yrs living in rural communities in tropical Ecuador. Asthma cases were selected based on the presence of recent wheeze and controls as a random sample of those without symptoms by questionnaire. Atopy was measured either by the presence of allergen specific IgE (asIgE) in serum or by allergen skin test reactivity (SPT). Overweight children were those with a Body Mass Index (BMI) $\geq+1$ z-score based on WHO growth curves (2007). Results Comparing atopic vs non-atopic children, the prevalence of SPT (adj. OR 2.12, 95\% CI 1.22 to 3.68) and the presence of asIgE (adj. OR 2.30, 95\% CI 1.10 to 4.83) was greater in overweight children compared to those with normal weight/underweight. Comparing non-atopic wheezers with non-atopic non-wheezers, being overweight was significantly associated with non-atopic wheeze (adj. OR 2.22, 95\% CI 1.07 to 4.63) when atopy was defined as asIgE but not SPT. Being overweight was not significantly associated with atopic wheeze (comparing atopic wheezers with atopic non-wheezers) and with severe wheeze or exercise-induced bronchospasm irrespective of atopic status.

Conclusion Although being overweight was associated with atopy, it was also associated with wheeze, and somewhat surprisingly this latter effect was not observed among atopic children.

\section{P2-199 ASSOCIATION BETWEEN ADHERENCE TO THE MEDITERRANEAN DIET AND BONE QUALITY IN A SAMPLE OF PORTUGUESE ADOLESCENTS}

doi:10.1136/jech.2011.142976j.33

\author{
${ }^{1,2} \mathrm{~T}$ Monjardino, ${ }^{* 1,2} \mathrm{R}$ Lucas, ${ }^{1,2}$ E Ramos, ${ }^{1,2} \mathrm{H}$ Barros. Institute of Public Health of the \\ University of Porto, Porto, Portugal; ${ }^{2}$ University of Porto Medical School, Porto, Portugal
}

Introduction Dietary patterns provide insights into how diet, rather than specific nutrients, affects bone health. We aimed to evaluate whether Mediterranean diet associates with bone mineral density at 13 years-old.

Methods We used data from 1232 adolescents (44.7\% males) born in 1990 and assessed at 13 years-old (EPITeen cohort). Adolescents were evaluated through physical examination, including height, weight and forearm bone mineral density (BMD) using dual-energy x-ray absorptiometry. Dietary intake was assessed using a food frequency questionnaire and adherence to the Mediterranean diet was evaluated through an adapted score (KIDMED index). The final score, the sum of all items, was classified into three adherence levels: -2 to 3,4 to 6 and $\geq 7$. The association between KIDMED index and $\mathrm{BMD}$ was quantified using linear regression. Coefficients were adjusted for body mass index, physical activity, smoking status and parental formal education.

Results Low KIDMED index was found in $23.9 \%$ of the girls and in $22.5 \%$ of the boys and $47.3 \%$ of girls and $46.1 \%$ of boys had intermediate index results. Mean (SD) BMD was $0.361(0.058) \mathrm{g} / \mathrm{cm}^{2}$ in girls and $0.344(0.051) \mathrm{g} / \mathrm{cm}^{2}$ in boys. Adherence to the Mediterranean diet showed no relation with $\mathrm{BMD}$ in girls. Significantly higher average $\mathrm{BMD}$ was found among boys with intermediate (0.013, 95\% CI: 0.003 to 0.023$)$ and high KIDMED index (0.017, 95\% CI 0.006 to 0.028 ) when compared to those with low index.

Conclusion A Mediterranean dietary pattern can be associated to better bone health since early in life.

\section{P2-200 POPULATION TRENDS IN THE INCIDENCE AND OUTCOMES OF ACUTE MYOCARDIAL INFARCTION IN A MEDITERRANEAN REGION OF SOUTHERN EUROPE}

doi:10.1136/jech.2011.142976j.33a

${ }^{1} \mathrm{C}$ Moreno-lribas, ${ }^{2} \mathrm{M}$ Imizcoz, ${ }^{1} \mathrm{M}$ Guevara, ${ }^{1} \mathrm{~J}$ Delfrade, ${ }^{3} \mathrm{~N}$ Alvarez-Arruti. ${ }^{*}$ Public Health Institute of Navarra, Pamplona-Iruña, Spain; ${ }^{2}$ Hospital Complex of Navarra, Pamplona-Iruña, Spain; ${ }^{3}$ Navarra Health Service, Pamplona-Iruña, Spain

Introduction The aim of this population-based study was to examine trends in the myocardial infarction incidence and mortality rates between 2000 and 2009 in Navarre, Spain.

Methods All admissions for myocardial infarction in a region of over half a million inhabitants from January 2000 to December 2009 were identified from the hospitals discharge databases. Age- and sexadjusted incidence and 30-day and 30-365-day mortality rates were calculated.

Results We identified 4451 persons 30 years of age or older with a first myocardial infarction. The incidence showed a significant decrease from 128 cases per 100000 person-years in 2000 to 98 cases per 100000 person-years in 2009, a 23\% relative decrease. Only in the case of ST-segment elevation myocardial infarction the decrease was significant. The proportion of patients who underwent revascularisation within 30 days after myocardial infarction increased from $26.8 \%$ in 2000 to $65.6 \%$ in 2009 . The age- and sex-adjusted 30 day mortality after myocardial infarction decreased from $10.7 \%$ in 2000-2004 to $7.6 \%$ in $2005-2009$. No changes were observed in 30-365-day mortality.

Discussion The important fall in the incidence observed in this population is consistent with other studies from other industrialised areas of the World. The higher rates of ST-segment elevation myocardial infarction vs non-ST could be indicating that the new definition is not broadly applied. The decreasing incidence, particularly ST-segment elevation myocardial infarction, is probably explained, at least in part, by substantial improvements in primary prevention efforts. Important improvements were observed also in the hospital care, particularly the big increase in revascularisation.

\section{P2-201 CHANGES IN AMINOTRANSFERASES INDICATE CHANGES IN HEPATOSTEATOSIS IN PEOPLE WITH TYPE 2 DIABETES: THE EDINBURGH TYPE 2 DIABETES STUDY}

doi:10.1136/jech.2011.142976j.34

${ }^{1} \mathrm{~J}$ Morling, ${ }^{*}{ }^{2} \mathrm{R}$ Williamson, ${ }^{2} \mathrm{M}$ Strachan, ${ }^{1} \mathrm{~J}$ Price, ${ }^{2} \mathrm{~S}$ Glancy, ${ }^{2} \mathrm{~L}$ Nee. ${ }^{1}$ University of Edinburgh, Edinburgh, UK; ${ }^{2}$ Western General Hospital, Edinburgh, UK

Background Recent data have suggested that plasma aminotransferases may be of limited use in the diagnosis of non-alcoholic 